See discussions, stats, and author profiles for this publication at: https://www.researchgate.net/publication/233922284

The primitive model of ionic fluids near its critical point in the PoissonBoltzmann and modified Poisson-Boltzmann theories

Article in The Journal of Chemical Physics · June 1994

DOI: $10.1063 / 1.466774$

\section{CITATIONS}

19

4 authors, including:

Lutful Bari Bhuiyan

University of Puerto Rico at Rio Piedras

149 PUBLICATIONS 2,559 CITATIONS

SEE PROFILE

A) Miguel Molero

Universidad de Sevilla

44 PUBLICATIONS 490 CITATIONS

SEE PROFILE

Some of the authors of this publication are also working on these related projects:

Project Linear Response Theory View project

Project 1. Electric double layer theory. 2. Electrolyte solution theory. View project
READS

47

Christopher W Outhwaite

120 PUBLICATIONS 2,796 CITATIONS

SEE PROFILE 


\title{
The primitive model of ionic fluids near its critical point in the Poisson-Boltzmann and modified Poisson-Boltzmann theories
}

\author{
L. B. Bhuiyan \\ Department of Physics, University of Puerto Rico, Rio Piedras, Puerto Rico 00931 \\ C. W. Outhwaite \\ School of Mathematics, University of Sheffield, Sheffield S3 7RH, England \\ M. Molero \\ Departamento de Quimica Fisica, Universidad de Sevilla, 41071 Sevilla, Spain \\ E. González-Tovar \\ Departamento de Física, Universidad Autónoma Metropolitana-Iztapalapa, Apartado Postal 55-534, \\ 09340 México, D.F. México
}

(Received 17 November 1993; accepted 22 February 1994)

\begin{abstract}
The Poisson-Boltzmann (PB) and modified Poisson-Boltzmann (MPB) theories are used to investigate the primitive model of ionic fluids in the low density-large coupling regime where the liquid-vapor transition is situated. The PB and MPB spinodal curves for the restricted primitive model are calculated from the virial route and compared with those from the mean spherical approximation (energy route) and the hybrid hypernetted-chain/mean spherical approximation (virial route). The effect of unequal ion sizes on the critical point and spinodal curves is also considered.
\end{abstract}

\section{INTRODUCTION}

A great deal of attention has recently been focused on the liquid-vapor coexistence region of ionic fluids. The most utilized model to represent an ionic fluid is the restricted primitive model (RPM) which is a system of equi-sized charged hard spheres moving in a uniform dielectric medium. This model can be applied to describe electrolyte solutions, and molten salts when the dielectric is ignored. The critical region is a very challenging theoretical problem due to the long-range electrostatic forces and its understanding is relevant to experimental work.

The existence of a coexistence curve was sketched by Stillinger and Lovett ${ }^{1}$ and some early Monte Carlo (MC) work carried out by Vorontsov-Vel'yaminov et $a l^{2}{ }^{2}$ The impetus for further theoretical work came from Stell et al. ${ }^{3}$ who gave estimates of the critical point using three different techniques, and the MC simulations of Larsen. ${ }^{4}$ Since then various theoretical estimates have been given for the critical point, the spinodal and coexistence curves, and structural behavior. Initial work concentrated on a combination of the classical Debye-Hückel (DH) and Bjerrum theories ${ }^{5,6}$ and this has recently been reconsidered by Fisher and Levin.? The ion pairing concept of Bjerrum motivated Gillan ${ }^{8}$ to use a cluster equilibrium theory and this has been improved by Pitzer and Schreiber. ${ }^{9}$ The promising application of integral equations based on the Ornstein-Zernike equation has been mainly restricted to the analytical mean spherical approximation (MSA) and related theories. ${ }^{6,10,11}$ This is because the potentially accurate hypernetted-chain (HNC) equation cannot be solved in the coexistence region. ${ }^{12,13}$ In contrast the hybrid HNC/MSA equation has been successfully applied to determine the spinodal curve and structure. ${ }^{14} \mathrm{~A}$ problem with the majority of approximate theories is the lack of consistency in the calculation of the thermodynamic properties via the various routes. In principle the compressibility equation provides a direct mechanism for determining the coexistence region but the lack of long-range correlations in the integral equation distribution functions flaws this approach. The alternatives are the virial or energy routes and the energy route is the most accurate for the MSA theory.

An alternative approach to the integral equations is that of the modified Poisson-Boltzmann (MPB) theory. ${ }^{15}$ This theory embeds the classical mean electrostatic approach of $\mathrm{DH}$ in the Bogoliubov-Born-Green-Kirkwood-Yvon integral equation hierarchies and provides a mechanism for correcting the approximations in the Poisson-Boltzmann (PB) theory. The hierarchy of equations are closed by replacing the departure of linear superposition of the potential of mean force of two ions by the corresponding fluctuation potential energy. ${ }^{16}$ An approximate fluctuation potential is then calculated and hence an improved radial distribution function derived in terms of the conditional mean electrostatic potentials about the ion species. The resulting "modified" PB equations are straightforward to solve numerically as the mean electrostatic potentials are screened in the liquid phase so that there are no convergence problems associated with the long-range Coulomb forces. Until recently the PB and MPB theories were restricted to the symmetric valency RPM. However, this restriction has now been removed and the MPB theory has proved to be very successful in describing the electrolyte regime. ${ }^{17}$ Because of this success, and the relative success of theories based on the classical $\mathrm{DH}$ and Bjerrum ideas, we expect the PB and MPB theories to provide a useful analysis of the liquid-vapor region. We find that in this region the PB and MPB theories are of comparable accuracy to the HNC/MSA equation for the RPM and we also briefly consider the PM for unequal ion sizes. 


\section{THEORY}

The PM pair potential $u_{i j}(r)$ for two ions $i$ and $j$ a distance $r$ apart is

$$
u_{i j}(r)=\left\{\begin{array}{l}
\infty, \quad r<a_{i j} \\
e^{2} z_{1} z_{j} / \epsilon r, \quad r>a_{i j}
\end{array},\right.
$$

where an ion of species $s$ has a radius $a_{s}$ and charge $z_{s}|e|, z_{s}$ valence, $a_{i j}=a_{i}+a_{j}$ and $\epsilon$ is the dielectric constant.

In the case of a binary electrolyte with equal ion sizes $\left(a_{i}=a_{j}=a / 2\right)$ and equal valences $\left(\left|z_{i}\right|=\left|z_{j}\right|=z\right)$, the MPB radial distribution function $g_{i j}(r)$ from the Kirkwood hierarchy is ${ }^{15}$

$$
g_{i j}(r)=\zeta_{i j}(r) \exp \left[-\left(z_{j} / z\right) L(v)\right],
$$

where

$$
\begin{aligned}
& \begin{aligned}
\zeta_{i j}(r)= & g_{i j}\left(e_{j}=0\right), \\
L(v)= & {[2(1+y) r]^{-1}[v(r+a)+v(r-a)} \\
& \left.+\kappa \int_{r-a}^{r+a} v(R) d R\right]
\end{aligned} \\
& \kappa^{2}=(4 \pi \beta / \epsilon) \sum_{s} \rho_{s} e^{2} z_{s}^{2}, \\
& v(r)=z|e| \beta r \psi_{i}(r)
\end{aligned}
$$

with $y=\kappa a$ and $\beta=1 / k T$. Here $\zeta_{i j}(r)$ is the exclusion volume term, $\psi_{i}(r)$ is the mean electrostatic potential a distance $r$ from an ion $i, \rho_{s}$ is the mean number density of ions of species $s$, and the sum in $s$ is over the ion species.

The mean electrostatic potential $\psi_{i}(r)$ satisfies Poisson's equation

$$
\nabla^{2} \psi_{i}(r)=-(4 \pi / \epsilon) \sum_{s}|e| z_{s} \rho_{s} g_{i s}(r)
$$

With the radial distribution function given by Eq. (2), the Poisson equation for the binary, symmetric valence RPM becomes

$$
\begin{array}{ll}
v^{\prime \prime}(r)=\kappa^{2} r \zeta(r) \sinh [L(v)], & r>a, \\
v(r)=v(a)-(a-r) v^{\prime}(a), & 0 \leqslant r \leqslant a .
\end{array}
$$

The classical PB equation follows from Eq. (5a) on putting $\zeta=1$ and $L(v)=v / r$, i.e., putting $a=0$ in $L[(v)]$. Various expressions have been considered for the exclusion volume term. We shall consider the three approximations:

$$
\begin{aligned}
\zeta(r)= & 1-\left[\epsilon \kappa^{2} /\left(4 \beta z^{2} e^{2}\right)\right]\left\{r^{-1} \int_{\max (a, r-a)}^{r+a} R \zeta(R)\right. \\
& \left.\times\left[a^{2}-(r-R)^{2}\right] \cosh [L(v)] d R-4 a^{3} / 3\right\}, \\
& r>a .
\end{aligned}
$$

(B)

$$
\begin{aligned}
\zeta(r)= & \exp \left\{\pi \int _ { r } ^ { \infty } \sum _ { t } \rho _ { t } \int _ { \operatorname { m a x } ( a , r - a ) } ^ { r + a } ( X / x ^ { 2 } ) \left(X^{2}-x^{2}\right.\right. \\
& \left.\left.-a^{2}\right) g_{i t}(X) \exp \left[-\beta e_{t} \Phi(x, X)\right] d X d x\right\} \\
& r>a
\end{aligned}
$$

where

$$
\Phi=[(1+y) 4 \pi a]^{-1} \int_{V^{*}} \nabla^{2} \psi d V
$$

is the fluctuation potential on the exclusion surface of a discharged ion, with $V^{*}$ the exclusion volume.

(C) $\zeta(r)=g^{\mathrm{PY}}(r)$,

the Percus-Yevick uncharged hard sphere radial distribution function.

The first approximation (A) corresponds to a first-order density expansion and is denoted by MPB4 in the literature ${ }^{15}$ while (B) gives the MPB5 theory and is derived from the Bogoliubov-Born-Green-Yvon hierarchy. At low concentrations the approximation (C) overestimates $\zeta(r)$ near contact because of the neglect of ion correlations. However, in most situations in the electrolyte solution regime this has a negligible effect on the thermodynamic and structural properties.

Equation (2) for $g_{i j}(r)$ is only symmetric under the interchange of ions $i$ and $j$ for the symmetric valence RPM. A procedure for deriving a symmetric version has recently been proposed $^{17}$ which gives

$$
\begin{aligned}
g_{i j}(r)= & \zeta_{i j}(r) \exp \left[-(\beta e / 2)\left\{z_{i}\left[L_{i}\left(u_{j}\right)+L_{i}^{0}\left(u_{j}\right)\right]\right.\right. \\
& \left.\left.+z_{j}\left[L_{j}\left(u_{i}\right)+L_{j}^{0}\left(u_{i}\right)\right]\right\}\right],
\end{aligned}
$$

where

$$
\begin{aligned}
\zeta_{i j}(r)= & g_{i j}\left(z_{i}=z_{j}=0\right), \\
L_{t}\left(u_{s}\right)= & {\left[2 r\left(1+\kappa a_{i t}\right)\right]^{-1}\left[u_{s}\left(r+a_{i t}\right)+u_{s}\left(r-a_{i t}\right)\right.} \\
& \left.+\kappa \int_{r-a_{i t}}^{r+a_{i t}} u_{s}(R) d R\right], \\
L_{t}^{0}\left(u_{s}\right)= & L_{t}\left(u_{s} \mid z_{s}=0\right),
\end{aligned}
$$

and $e=|e| u_{s}=r \psi_{s}$. In Eq. (10b), the ion $i$ is the species with the smallest radius. For ions of equal size the potentials about the discharged ions $\psi_{s}^{0}$ are zero. A symmetrical version of the PB theory (SPB) now follows on putting $L_{t}\left(u_{s}\right)=\psi_{s}$ and $L_{t}^{0}\left(u_{s}\right)=\psi_{s}^{0}$. When the ions are of different size and/or valence we now have for a binary electrolyte a pair of coupled equations to solve for the mean electrostatic potentials of the two ion species. The PB and SPB theories we treat here will always include the estimate $(C)$ for the exclusion volume term. An alternative symmetric $g_{i j}(r)$ was used in Ref. 17 which is given by replacing $L_{i}\left(u_{j}\right)$ by $L_{j}\left(u_{j}\right)$ and $L_{j}\left(u_{i}\right)$ by $L_{i}\left(u_{i}\right)$ in Eq. (9). Equation (9) is formally more correct but both theories are of comparable accuracy when compared with the unequal size electrolyte MC simulations. 

by

The spinodal line for the gas-liquid transition is given

$$
\left(\frac{\partial p}{\partial \rho}\right)_{T}=0,
$$

where $\rho=\Sigma_{s} \rho_{s}$ and $p$ is the pressure. We determine the values of $\rho$ and $T$ for which Eq. (11) holds by using $p$ given by the virial equation ${ }^{15}$

$$
\beta p / \rho=1+(2 \pi / 3 \rho) \sum_{s} \sum_{t} \rho_{s} \rho_{t} g_{s t}\left(a_{s t}\right) a_{s t}^{3}+\beta E / 3 \rho,
$$

where the internal energy $E$ is given by

$$
E=(1 / 2) \sum_{s} e z_{s} \rho_{s}\left[\psi_{s}\left(a_{i s}\right)-e z_{s} / \epsilon a_{i s}\right]
$$

An apparent direct method would be to use the symmetric valence RPM compressibility relation

$$
\begin{aligned}
\beta\left(\frac{\partial p}{\partial \rho}\right)_{T} & =1-(\rho / 2) \int\left(c_{++}+c_{+-}\right) d \mathbf{r} \\
& =\left[1+\rho \int h_{s}(r) d \mathbf{r}\right]^{-1}
\end{aligned}
$$

where $c_{i j}$ is the direct correlation function and $h_{s}=\left(g_{++}+g_{+-}-2\right) / 2$. However, as found in the $\mathrm{HNC} /$ MSA and MSA equations, ${ }^{10,14}$ the necessary long-range behavior in $h_{s}$ is not present in the MPB equation to make the right-hand side of Eq. (14) vanish.

The virial equation provides a route for constructing approximate analytical theories on stipulating $g_{i j}$ and $\psi_{s}$ at contact. We suggest two analytical theories for the RPM based on results from the electrolyte regime. The only two realistic analytical theories are the DH and MSA theories. The MSA, and to a lesser extent the DH theory, both predict reasonable estimates for the internal energy $E\left[\right.$ i.e., $\psi_{s}(a)$ ]. However both suffer from underestimating $g_{i j}(a)$, and indeed, under certain conditions $g_{i j}(a)$ for like ions can be negative. A fairly successful strategy to obtain an improved analytical $g_{i j}(r)$ is to use an exponential version. Versions which have been considered are the $\mathrm{DHX}^{18}$ and $\mathrm{MSX}^{19}$ theories which are derived from the DH and MSA theories, respectively. Considering only the DHX theory we have, at contact,

$$
g_{i j}^{\mathrm{DHX}}(a)=\exp \left[\beta z_{i} z_{j} e^{2} / \epsilon(1+y)\right] .
$$

So using the DH $\psi_{s}(a)$, we have from Eq. (12) the $\mathrm{DHX}+\mathrm{DH}$ expression for the pressure

$$
\begin{aligned}
\beta p / \rho= & 1+(2 \pi / 3 \rho) \sum_{s} \sum_{t} \rho_{s} \rho_{t} g_{s t}^{\mathrm{DHX}}(a) a_{s t}^{3} \\
& -y^{3} /\left[24(1+y) \pi \rho a^{3}\right]
\end{aligned}
$$

and using the MSA $\psi_{s}(a)$, we have the DHX+MSA expression

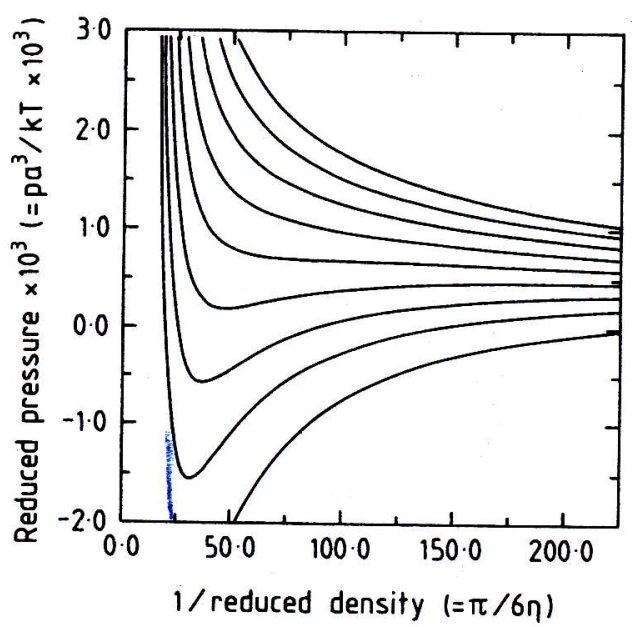

FIG. 1. MPB(C) reduced pressure isotherms for $2100 \mathrm{~K} \leqslant T \leqslant 2950 \mathrm{~K}$. Bottom curve $T=2100 \mathrm{~K}$, next curve $T=2250 \mathrm{~K}$ and remaining curves increasing in intervals of $100 \mathrm{~K}$.

$$
\begin{aligned}
\beta p / \rho= & 1+(2 \pi / 3 \rho) \sum_{s} \sum_{t} \rho_{s} \rho_{t} g_{s t}^{\mathrm{DHX}}(a) a_{s t}^{3} \\
& -\left[1+y-(1+2 y)^{1 / 2}\right] y /\left(12 \pi \rho a^{3}\right) .
\end{aligned}
$$

\section{RESULTS}

The PB and MPB equations for the mean electrostatic potentials were solved by a previously developed quasilinearization technique ${ }^{20,21}$ with the PY hard sphere $g_{i j}$, used in the expression (C) for $\zeta$, determined by Perram's technique. ${ }^{22}$ Having calculated $\psi_{i}$ and $\zeta$ for the RPM, the radial distribution function follows from Eq. (2). This technique is very robust and requires few iterations for convergence. It has also proved successful in solving a related MPB equation in electric double layer theory. ${ }^{23}$

The thermodynamic properties for the binary PM depend on the dimensionless parameters

$$
\eta=(\pi / 6) \sum_{s} \rho_{s}\left(2 a_{s}\right)^{3}, \quad \Gamma=e^{2} \beta / \epsilon a,
$$

where $a=a_{i}+a_{j}, \eta$ is the packing fraction, and $\Gamma$ the inverse reduced temperature or the plasma coupling parameter (for $\epsilon=1$ ). A third dependent dimensionless quantity is

$$
y=\kappa a=\sqrt{ }\left[3 z_{i} z_{j} \eta \Gamma(\alpha+1)^{3}\left(z_{i}-z_{j}\right) /\left(\alpha^{3} z_{j}-z_{i}\right)\right],
$$

where $\alpha=a_{i} / a_{j}, a_{i} \leqslant a_{j}$ being the ion size ratio for the unequal ion size case. In all the calculations $a$ was taken to be $4.2 \mathrm{~A}, z_{i}=-z_{j}=1$, and $\eta$ and $\Gamma$ varied. For unequal ion sizes $\alpha$ was 0.8 or 0.5 with again $a=a_{i}+a_{j}=4.2 \mathrm{~A}$. When the ion sizes are unequal a pair of coupled equations have to be solved for the two mean electrostatic potentials $\psi_{i}, \psi_{j}$ corresponding to the two species $i$ and $j$. The exclusion volume term is then only treated using $(\mathrm{C})$ and the radial distribution function determined from Eq. (9).

The MPB (C) restricted PM isotherms in Fig. 1 of the reduced pressure $p^{*}\left(=p \beta a^{3}\right)$ against the inverse reduced density $1 / \rho^{*}\left(=1 / \rho a^{3}=\pi / 6 \eta\right)$ for $18.95 \geqslant \Gamma \geqslant 13.49$ 


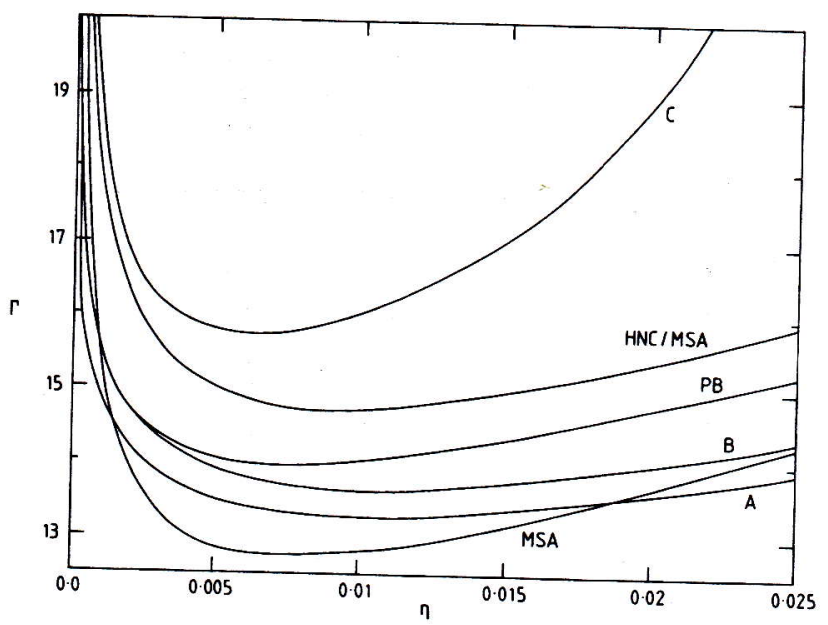

FIG. 2. Spinodal curves for the three MPB versions (A, B, C), the PB, HNC/MSA, and MSA. All the curves, except that for the MSA, are derived via the pressure equation.

( $2100 \mathrm{~K} \leqslant T \leqslant 2950 \mathrm{~K}$, for $\epsilon=1$ ) show van der Waals loops which indicate the presence of a phase transition. Analogous van der Waals loops were found for the PB theory and the (A) and (B) versions of the MPB theory. The stationary points of the loops were tracked numerically and gave the spinodal curves shown in Fig. 2. Also in Fig. 2 are the curves for the HNC/MSA theory from the virial route and the MSA from the energy route. The qualitative shapes of all the curves are similar except for the MPB (C) and the MSA curves which vary more rapidly with increasing density above their critical density. Estimates of the critical point values of $\eta_{c}$ and $\Gamma_{c}$ for the MPB and PB theories are given by their values for which the maxima and minima of the van der Waals loops coalesce. These critical values are shown in Table I for the RPM along with some other theories plus those of the DHX + DH and DHX +MSA, Eqs. (16) and (17). As seen in the table, a wide range of critical values have

TABLE I. Comparison of the RPM critical parameters predicted by various theories. The subscripts $v$ and $c$ on the HNC/MSA theory denotes the virial and compressibility route, respectively, while the MSA values are calculated from the energy route. The virial route is used to calculate the PB, MPB, $\mathrm{DHX}+\mathrm{DH}$, and DHX + MSA critical values.

\begin{tabular}{llll}
\hline \hline \multicolumn{1}{c}{ Theory } & \multicolumn{1}{c}{$\Gamma_{c}$} & $\eta_{c}$ & $(\kappa a)_{c}$ \\
\hline MC (Ref. 24) & 14.2 & 0.037 & 3.54 \\
MC (Ref. 25) & 17.9 & 0.021 & 3.00 \\
MPB(A) & 13.28 & 0.010 & 1.78 \\
MPB(B) & 13.64 & 0.010 & 1.81 \\
MPB(C) & 15.74 & 0.0062 & 1.53 \\
PB & 13.97 & 0.0073 & 1.57 \\
HNC/MSA & 14.7 & 0.0085 & 1.73 \\
(Ref. 14) & & & \\
HNC/MSA & 13.75 & 0.021 & 2.63 \\
(Ref. 14) & & & \\
EGM (Ref. 6) & 11.95 & 0.0094 & 1.64 \\
PS (Ref. 9) & 14.7 & 0.026 & 3.03 \\
MSA & 12.73 & 0.0076 & 1.52 \\
DHX+DH & 15.80 & 0.0031 & 1.08 \\
DHX+MSA & 15.72 & 0.0034 & 1.14 \\
\hline \hline
\end{tabular}

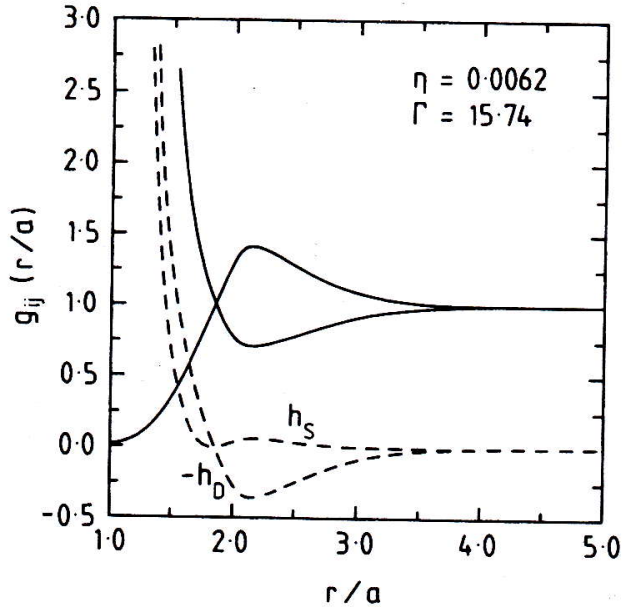

FIG. 3. The $M P B(C)$ radial distribution functions at the $M P B(C)$ critical point $\eta=0.0062, \Gamma=15.74$. The solid curve represents $g_{i j}$ and the dashed curves the sum $h_{s}=\left(g_{++}+g_{+-}-2\right) / 2$ and the difference $h_{d}=\left(g_{++}-g_{+-}\right) / 2$.

been predicted. Only recently have fairly reliable MC simulations $^{24,25}$ become available for comparison purposes. These MC results seem to indicate that $14<\Gamma_{c}<18$ and $0.02<\eta_{c}<0.04$. The PB, MPB, DHX + DH, and DHX +MSA all predict reasonable estimates for $\Gamma_{c}$ but give too low a value for the critical density. This is especially true for the $\mathrm{DHX}+\mathrm{DH}$ and DHX + MSA theories. The PB and MPB approaches are comparable in accuracy to that of the $\mathrm{HNCl}$ MSA theory, from the pressure equation, in predicting the critical point. Indeed, the $\mathrm{PB}$ critical point and spinodal curve is closer to that of the HNC/MSA theory than the MPB theory and we would thus expect a good estimate of the $\mathrm{HNC/MSA}$ coexistence curve from the pressure equation to be given by the corresponding $\mathrm{PB}$ coexistence curve.

The MPB and HNC/MSA radial distribution functions near the critical density are in very good agreement over the range of $\Gamma(4 \leqslant \Gamma \leqslant 14.7)$ in Figs. $3-5$ of Ref. 14 , and their agreement is reminiscent of that found between them in the electrolyte regime and the electric double layer for planar, ${ }^{23}$ spherical, $^{26}$ and cylindrical geometries. ${ }^{27}$ Unfortunately no MC structural results exist in the coexistence region to test the accuracy of the two theories. Strong, damped, oscillations occur in the MPB and HNC/MSA $g_{i j}(r)$ as the coupling parameter $\Gamma$ increases. However, as the critical region is approached the expected long-range correlations do not occur in the $h_{s}(r)$ profiles in either the MPB, Fig. 3, or $\mathrm{HNC/MSA}$ theories. This is the reason why the compressibility equation cannot be used to investigate the critical phenomena. A strategy to introduce long-range behavior into $h_{s}(r)$ in the HNC/MSA theory through the choice of $c_{i j}$ 's to satisfy the compressibilty relation [Eq. (14)] has been given by González-Tovar et al. ${ }^{14}$ Charge density oscillations, which arise because of charge exclusion in an ions' atmosphere due to the ions in the atmosphere having size, occurs in the mean electrostatic potential of the MPB at $y=1.2412^{15}$ and in the MSA at $y=1.229 .^{28}$ Stillinger and Lovett ${ }^{1}$ predicted charge oscillations for $y>\sqrt{ } 6$ by considering their sec- 
TABLE II. Comparison of the unequal radii PM critical parameters predicted by the MPB $(C)$, SPB, and MSA theories. The MPB(C) and SPB values are calculated via the virial route and the MSA values from the energy route.

\begin{tabular}{lccccccc}
\hline \hline & \multicolumn{4}{c}{$\alpha=0.5$} & & \multicolumn{3}{c}{$\alpha=0.8$} \\
\cline { 2 - 3 } \cline { 6 - 8 } Theory & $\Gamma_{c}$ & $\eta_{c}$ & $(\kappa a)_{c}$ & & $\Gamma_{c}$ & $\eta_{c}$ & $(\kappa a)_{c}$ \\
\hline MPB(C) & 14.54 & 0.0099 & 1.61 & & 15.12 & 0.0069 & 1.55 \\
SPB & 14.11 & 0.0083 & 1.42 & & 13.94 & 0.0075 & 1.56 \\
MSA $_{e}$ & 12.11 & 0.0114 & 1.58 & & 12.66 & 0.0080 & 1.53 \\
\hline \hline
\end{tabular}

ond moment condition with the neutrality condition. These damped oscillations seem to be the harbinger of the necessary long-range behavior in $h_{s}(r)$ as the coexistence curve is approached from the liquid phase. The association of the onset of charge-density oscillations with the coexistence curve was noted by Stell et $a .^{3}$ and Larsen. ${ }^{4}$ Present MC estimates of the critical value of $y$ are much higher than the MPB and MSA theories, Table I. Interestingly new MC work $^{29}$ has indicated that the Stillinger-Lovett relation does not appear to hold in the vapor region and this is supported by the MPB calculations. Although the MPB does not satisfy the Stillinger-Lovett relation exactly, it is always nearly satisfied above the critical temperature but in the coexistence region no correspondence is observable. No charge oscillations are predicted by the PB theory while the $\mathrm{PB} g_{i j}(r)$ are only in qualitative agreement with the MPB and HNC/MSA theories for both $\Gamma$ and $\eta$ being small.

A simple generalization of the PM is to consider unequal ion sizes. This has been considered for the $\mathrm{MPB}(\mathrm{C})$, SPB, and MSA theories and the critical value of the parameters given in Table II for $\alpha=0.8$ and 0.5 with $a=4.2 \mathrm{~A}$. Both the

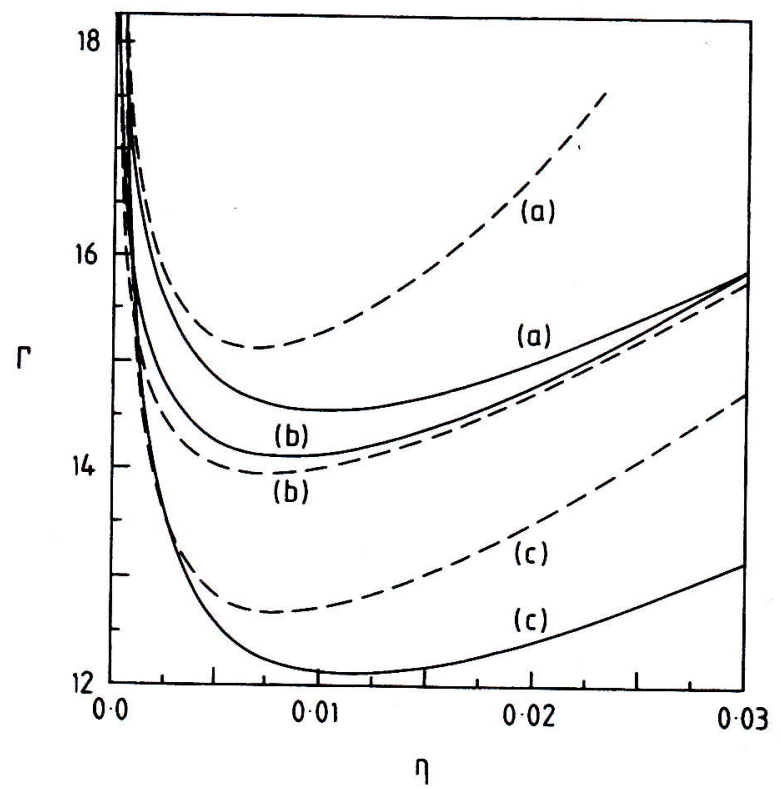

FIG. 4. Spinodal curves for (a) MPB(C), (b) SPB, and (c) MSA at different ionic radii. Dashed line $\alpha=0.8$, solid line $\alpha=0.5$. The MPB and SPB curves are calculated from the pressure equation and the MSA from the energy equation.

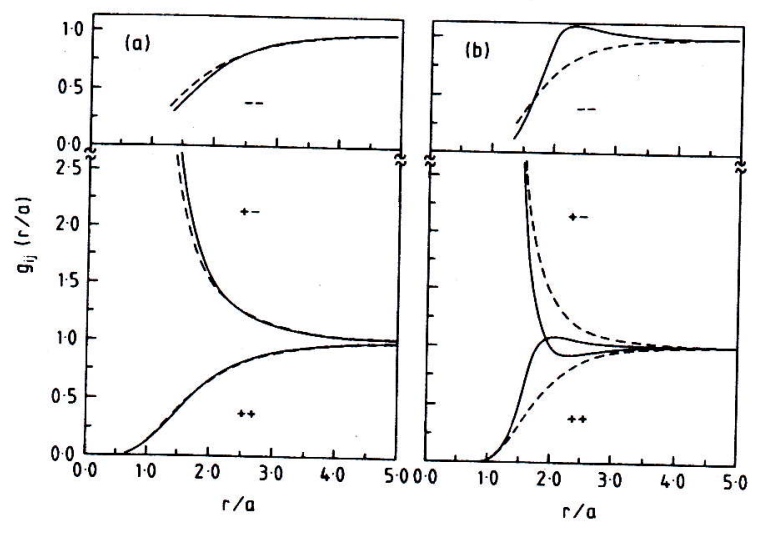

FIG. 5. MPB(C) and SPB radial distribution functions at $\alpha=0.5$. The solid curves are the MPB and the dashed curves the SPB. (a) is at $\eta=0.01, \Gamma=4$. (b) is at the respective critical points of the MPB and SPB theories.

MPB and MSA theories indicate an increase in the critical temperature and density as the ionic radii ratio decreases from unity. Surprisingly the critical temperature of the SPB theory initially rises then falls as $\alpha$ decreases which indicates a possible failing of the theory. The corresponding spinodal curves are given in Fig. 4 where both the MPB and MSA predict an increase in $T$ for the coexistence curve, as $\alpha$ decreases, at densities near and above the critical point. Thus the MPB and MSA theories suggest that for a given density the coexistence region increases with an increase in ion size variation. The SPB and MPB radial distribution functions in Fig. 5 for $\alpha=0.5$ show a behavior analogous to the equal ion size case although the damped charge-density oscillations at the MPB critical point are not so pronounced. Again, no long-range behavior is observed in the correlation functions in the critical region.

\section{CONCLUSIONS}

The PB and MPB theories provide an appealing alternative approach to the HNC/MSA and MSA integral equations for treating the liquid-vapor critical region. Indeed the HNC equation cannot be solved at low densities in the temperature range of interest. In contrast the theories based on the mean electrostatic potential can easily be solved numerically, there being no convergence problems at the low densities. Again it is straightforward to extend the potential based theories to treat unequal ion sizes and ionic mixtures. A remarkable feature of the PB thermodynamic predictions for the PM is their close agreement with those of the supposedly more accurate MPB, MSA, and HNC/MSA equations. This parallels the accurate osmotic coefficient predicted by the PB theory from the virial route in the electrolyte regime. ${ }^{17}$ The relative success of the PB theory and its relatively straightforward numerical solution indicates that it might be feasible, and of interest, to determine its coexistence curve using the Maxwell construction.

\section{ACKNOWLEDGMENTS}

This work was partially supported by the NSF through Grant No. CHE-8907130. L. B. B. acknowledges an internal 
grant through FIPI, University of Puerto Rico, E. G.-T. the European Community through a Marie Curie fellowship and M. M. the Spanish D.G.I.C.Y.T. through Grant No. PB91/ 0609. We also thank Professor Marcelo Lozada-Cassou for supplying us with some HNC/MSA data and Dr. T. Das for computing help.

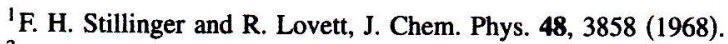

${ }^{2}$ P. N. Vorontsov-Vel'yaminov, A. M. El'yashevich, L. A. Morgenshtern, and V. P. Chasovskikh, High Temp. 8, 261 (1970).

${ }^{3}$ G. Stell, K. C. Wu, and B. Larsen, Phys. Rev. Lett. 37, 1369 (1976).

${ }^{4}$ B. Larsen, J. Chem. Phys. 65, 3431 (1976).

${ }^{5}$ H. L. Friedman and B. Larsen, J. Chem. Phys. 70, 92 (1979).

${ }^{6} \mathrm{~W}$. Ebling and M. Grigo, Ann. Phys. 37, 21 (1980).

${ }^{7}$ M. E. Fisher and Y. Levin, Phys. Rev. Lett. 71, 3826 (1993).

${ }^{8}$ M. J. Gillan, Mol. Phys. 49, 421 (1983).

${ }^{9}$ K. S. Pitzer and D. R. Schreiber, Mol. Phys. 60, 1067 (1987).

${ }^{10}$ M. Medina-Noyola and D. A. McQuarrie, J. Stat. Phys. 18, 445 (1978).

${ }^{11}$ M. Medina-Noyola, J. Chem. Phys. 81, 5059 (1984).

${ }^{12}$ J. S. Hoye, E. Lomba, and G. Stell, Mol. Phys. 75, 1217 (1992); 79, 523 (1993).
${ }^{13}$ L. Belloni, J. Chem. Phys. 98, 8080 (1993).

${ }^{14}$ E. González-Tovar, M. Lozada-Cassou, L. Mier-y-Terán, and M. MedinaNoyola, J. Chem. Phys. 95, 6784 (1991).

${ }^{15}$ C. W. Outhwaite, in Statistical Mechanics (The Chemical Society, London, 1975), Vol. 2, p. 188.

${ }^{16}$ C. W. Outhwaite, J. Chem. Phys. 50, 2277 (1969).

${ }^{17}$ C. W. Outhwaite, M. Molero, and L. B. Bhuiyan, J. Chem. Soc. Faraday Trans. 87, 3227 (1991); 89, 1315 (1993).

${ }^{18}$ D. N. Card and J. P. Valleau, J. Chem. Phys. 52, 6232 (1970).

${ }^{19} \mathrm{C}$. W. Outhwaite, Chem. Phys. Lett. 37, 383 (1976).

${ }^{20}$ D. M. Burley, V. C. L. Hutson, and C. W. Outhwaite, Mol. Phys. 23, 867 (1972); 27, 225 (1974).

${ }^{21}$ C. W. Outhwaite, J. Chem. Soc. Faraday Trans. 2 83, 949 (1987).

${ }^{22}$ J. W. Perram, Mol. Phys. 30, 1505 (1975).

${ }^{23}$ S. L. Carnie and G. M. Torrie, Adv. Chem. Phys. 56, 141 (1984).

${ }^{24}$ J. P. Valleau, J. Chem. Phys. 95, 584 (1991).

${ }^{25}$ A. Z. Panagiotopoulos, Fluid Phase Equil. 76, 97 (1992).

${ }^{26}$ L. Degreve, M. Lozada-Cassou, E. Sanchez, and E. González-Tovar, J. Chem. Phys. 98, 8905 (1993).

${ }^{27}$ L. B. Bhuiyan and C. W. Outhwaite, Philos. Mag. (in press).

${ }^{28}$ C. W. Outhwaite and V. C. L. Hutson, Mol. Phys. 29, 1521 (1975).

${ }^{29}$ J. M. Caillol, J. Chem. Phys. 100, 2161 (1994). 